\title{
Immunomodulatory Properties of PDLSC and Relevance to Periodontal Regeneration
}

\author{
Naohisa Wada $^{1}$ - Atsushi Tomokiyo ${ }^{2} \cdot$ Stan Gronthos $^{3,4} \cdot$ P. Mark Bartold ${ }^{5}$
}

Published online: 10 September 2015

(C) Springer International Publishing AG 2015

\begin{abstract}
Periodontitis, a major chronic inflammatory disease of dental tissue, causes periodontal tissue destruction and alveolar bone resorption. When large amounts of alveolar bone are lost, it is very difficult to regenerate the bone using conventional treatments. Periodontal ligament stem cells (PDLSCs) have attracted much attention as potential cell sources for alternative periodontitis regenerative treatment because of their capacity for self-renewal and multipotency. Recently, PDLSCs were found to possess immunomodulatory properties. PDLSCs lack the expression of immune costimulating factors and suppress immune cell proliferation following stimulation with mitogens or in mixed allogeneic
\end{abstract}

This article is part of the Topical Collection on Stem-Cell Biology for Tooth and Periodontal Regeneration

Naohisa Wada

wada@dent.kyushu-u.ac.jp

Atsushi Tomokiyo

tomokiyo@dent.kyushu-u.ac.jp

Stan Gronthos

stan.gronthos@adelaide.edu.au

P. Mark Bartold

mark.bartold@adelaide.edu.au

1 Division of General Dentistry, Kyushu University Hospital, Kyushu University, 3-1-1 Maidashi, Higashi-ku, Fukuoka 812-8582, Japan

2 Department of Endodontology and Operative Dentistry, Division of Oral Rehabilitation, Faculty of Dental Science, Kyushu University, 3-1-1 Maidashi, Higashi-ku, Fukuoka 812-8582, Japan

3 Mesenchymal Stem Cell Laboratory, School of Medicine, University of Adelaide, Adelaide, SA, Australia

4 South Australian Health and Medical Research Institute, Adelaide, SA, Australia

5 School of Dentistry, Colgate Australian Clinical Dental Research Centre, University of Adelaide, Adelaide, SA, Australia lymphocyte reactions. Because of these characteristics, the anti-inflammatory effects of PDLSCs and the establishment of allogeneic PDLSC transplantation therapies have been well studied. The aim of this review is to provide an assessment of the latest findings on the immunomodulatory properties of PDLSCs and their potential clinical application for periodontal tissue regeneration.

Keywords Periodontal regeneration $\cdot$ Periodontal ligament stem cells $\cdot$ Immunomodulatory property $\cdot$ Allogeneic cell transplantation · Transplant rejection · Autoimmune disease

\section{Introduction}

Periodontitis is a chronic inflammatory disease of the periodontal tissue. Periodontitis is caused by bacterial infection, resulting in an inflammatory reaction which leads to the destruction of periodontal tissue, including the periodontal ligament and alveolar bone and, in severe cases, tooth loss. The main approach for the treatment of periodontitis is the removal of dental plaque, which is composed of bacteria. More recently, regenerative treatments for damaged or destroyed periodontal tissue have achieved great progress through the use of barrier membranes, enamel matrix derivative, and recombinant proteins; these have become the mainstay of periodontal treatment [1-4]. However, the efficacy of all these approaches has been limited, because periodontal tissue regeneration is dependent on the presence of the recruitment and differentiation of local stem cells at the site of the damaged tissue. Thus, the development of alternative therapies using dental stem cell transplantation has been investigated as an approach to more efficiently regenerate damaged periodontal tissue. Given that periodontal tissue destruction occurs following bacterial infection and inflammation, the delivery of an 
exogenous cell source to site of damaged periodontal tissue may be more effective at inducing regeneration, while also exhibiting anti-inflammatory and immunosuppressive properties.

In 2000, Gronthos et al. first reported the isolation of dental pulp stem cells (DPSCs) from human dental pulp tissue as dental tissue mesenchymal stem cells (MSCs) [5]. Later, in 2004, periodontal ligament stem cells (PDLSCs) were isolated from human periodontal ligament [6]. Interestingly, although PDLSCs possessed the capacity for self-renewal and multipotential differentiation into osteogenic, adipogenic, and chondrogenic lineages in vitro similar to bone marrow MSCs (BMSCs) and DPSCs, they exhibited their own unique potential in vivo in contrast to other tissue-derived MSCs. When BMSCs, DPSCs, or PDLSCs were subcutaneously transplanted into the dorsal surfaces of immunocompromised mice with hydroxyapatite-tricalcium phosphate ceramic particles as a carrier, BMSCs formed bone-like tissue with bone marrow, DPSCs formed dentin/pulp complex-like tissue, and PDLSCs formed a cementum-like tissue layer with condensed collagen fibers like Sharpey's fibers, which resembled PDL structures. These observations indicate that different MSClike populations regenerate their tissue of origin in vivo, presumably based on epigenetic memory. Therefore, PDLSCs are an ideal cell source for the regeneration of periodontal tissue compared with MSCs derived from other dental or non-dental tissues.

PDLSCs have been reported to possess immunomodulatory properties similar to BMSCs $[7 \bullet \bullet]$. This property could be a valuable advantage for cell transplantation therapies as the inflammatory condition in periodontitis inhibits the natural repair processes involving local cells, including stem, progenitor, and mature cells. Chronic periodontitis represents an inflammatory response in the periodontal tissues, which is elicited by bacteria in dental plaque. The early/stable lesion of chronic periodontitis is dominated by $\mathrm{T}$ cells and macrophages, which are mediated by T-helper 1 (Th1) cytokines, including interleukin (IL)- 1 and interferon (IFN)- $\gamma$, while the advanced/ progressive lesion of chronic periodontitis is dominated by $\mathrm{B}$ cells and plasma cells, which are dependent on Th2 cytokines, including IL-4 [53]. This inflammation leads to alveolar bone loss caused by excessive activation of osteoclasts [54]. When alveolar bone is extensively resorbed, it is difficult to regenerate bone volume to normal levels owing to quantitative limitations and reduced regenerative capacity caused by inflammation. Thus, to effectively regenerate damaged periodontal tissue, including alveolar bone, the ideal therapy must include both regenerative and anti-inflammatory/immunosuppressive approaches. In this review, we describe the potential of PDLSCs as a cell source for periodontal tissue engineering, with a focus on their immunomodulatory properties.

\section{Immunomodulatory Properties of MSCs}

In recent decades, MSCs have been widely studied as potential cell therapy agents for transplant rejection and immunemediated diseases because of their immunomodulatory properties, as demonstrated in numerous pre-clinical animal studies and in human clinical trials. MSCs express very low levels of major histocompatibility complex (MHC) class II antigens and costimulatory molecules, such as CD40, CD80, and CD86 [8]. They exert their immunomodulatory function on various immune cells including T cells, B cells, natural killer cells, monocytes, macrophages, dendritic cells, and neutrophils via several mechanisms [9-14]. The key immunomodulatory factors and cytokines expressed by MSCs have been reported to include transforming growth factor-beta (TGF- $\beta$ ), hepatocyte growth factor (HGF), indoleamine 2, 3-dioxygenase (IDO), prostaglandin E2, nitric oxide, IL-4, IL-6, IL-10, IL-1 receptor antagonist, heme oxygenase-1, and human leukocyte antigen-G [15-22].

A number of studies have reported that the administration of allogeneic MSCs results in a marked suppression of host immune reactions in preclinical animal models of transplant rejection and immune-mediated diseases. Although transplant rejection or graft-versus-host disease (GVHD) is almost unavoidable during allogeneic transplantation, the administration of baboon MSCs in vivo before the replacement of third-party skin grafts led to prolonged skin graft survival in recipient baboons [23]. Transplantation of murine MSCs into murine GVHD models resulted in a reduction of GVHD severity [24], suggesting that allogeneic MSCs could be effective therapeutic agents in transplant rejection and GVHD. MSCs have also been reported to be effective in autoimmune diseases, such as systemic lupus erythematosus (SLE), rheumatoid arthritis, and multiple sclerosis, in preclinical animal models. In MRL/lpr mouse model of SLE-like disease, allogeneic bone marrow stromal cell transplantation recovered multiorgan dysfunction more effectively than administration of cyclophosphamide, which is a conventional immunosuppressive therapy [25]. In the collagen-induced arthritis mouse model, MSC injection prevented severe damage to bone and cartilage through inhibitory effects on $\mathrm{T}$ cell proliferation and inflammatory cytokine expression [26]. Moreover, in the experimental autoimmune encephalomyelitis (EAE) mouse model of multiple sclerosis, a chronic autoimmune, inflammatory, demyelinating, neurodegenerative disease of the central nervous system, systematic injection of MSCs alleviated EAE by suppressing infiltration of $\mathrm{T}$ cells into the brain [27, 28].

Based on the positive outcomes of their potential to suppress immune cell proliferation and function in in vitro and in vivo animal studies, MSCs have been proposed as a therapeutic option for the treatment of GVHD and autoimmune diseases by clinical application of human MSCs. The first clinical trial of allogeneic MSC transplantation was the 
transplantation of maternal-derived haploidentical MSCs into a patient with severe treatment-resistant grade IV acute GVHD of the gut and the liver, resulting in a rapid improvement of GVHD for up to 1 year post-transplantation [29]. A subsequent multicenter phase II clinical trial showed that 39 of 55 patients with steroid-resistant severe acute GVHD responded to treatment with MSCs, suggesting that MSC infusion might be an effective therapy for some patients with acute GVHD [30]. Furthermore, the use of cellular therapies to treat acute GVHD has been supported by a number of other clinical trials transplanting MSCs alone or in combination with allogeneic hematopoietic stem cells [31]. Clinical trials of allogeneic human MSC transplantation in patients with SLE have also shown promising results with improvements in symptoms $[32,33]$. The outcomes of these clinical trials indicate that novel therapies using allogeneic MSC transplantation are possible, although further large randomized double-blind clinical trials are necessary.

\section{Immunomodulatory Properties of Dental-Tissue-Derived Stem Cells}

In addition to DPSCs and PDLSCs, MSC populations have also been identified from several human dental tissues, including exfoliated deciduous teeth pulp tissue (SHED), apical papilla (SCAP), dental follicle stem cells (DFSCs) that are the cells of origin of the periodontal ligament, and gingiva (GMSCs) [5, 6, 34-37]. As these dental-tissue-derived stem cells also exhibit self-renewal and multilineage differentiation potentials, their capacities to regenerate functional dental tissues have been studied in vitro and in vivo. Moreover, dentaltissue-derived stem cells have recently been reported to possess potent immunomodulatory functions comparable with BMMSCs.

\section{PDLSCs}

In 2009, the potential of human PDLSCs to modulate immune function was first reported by Wada et al. [7••]. In an in vitro study, they demonstrated that human PDLSCs inhibited peripheral blood mononuclear cell (PBMNC) proliferation following mitogen stimulation or in an allogeneic mixed lymphocyte reaction similar to bone marrow stromal stem cells. These observations are supported by other studies demonstrating the immunomodulatory effects of canine PDLSCs and MSCs from the human periapical lesion, which partially originate from PDL cells, on PBMNCs [38, 39]. Human PDLSCs lacked the expression of immune costimulatory factors, such as MHC class II antigen, CD40, CD80, and CD86 [7••, 40••]. Moreover, IFN- $\gamma$, secreted by activated human PBMNCs, induced PDLSCs to secrete soluble factors, including TGF- $\beta$, HGF, and IDO, which partially suppressed PBMNC proliferation $\left[7^{\bullet \bullet}\right]$. Another potential mechanism of immunoregulation by PDLSCs was proposed via the secretion of prostaglandin E2 (PGE2) [40••, 41]. Human PDLSCs suppressed $\mathrm{T}$ cell proliferation mediated by $\mathrm{PGE} 2$, and $\mathrm{B}$ cell proliferation, differentiation, and migration mediated by programmed cell death protein 1 (PD-1) and its ligand (PD-L1) [40••, 42•]. Interestingly, Liu et al. reported that PDLSCs isolated from inflamed PDL tissue (inflamed PDLSCs) diminished the inhibitory effects on PBMNC proliferation and the induction of CD4+CD25+FOXP3+ regulatory $\mathrm{T}$ cell differentiation and IL-10 secretion compared with healthy PDLSCs [43]. Inflamed PDLSCs have also been reported to maintain their proliferative potential, multipotency, and regenerative capacity for cementum and PDL tissue [44]. This suggested that healthy PDLSCs, which fulfill both the regenerative and immunosuppressive requirements of an ideal transplant cell for periodontal tissue regeneration, could be a more appropriate cell source than inflamed PDLSCs. Given these features, the transplant of PDLSCs is expected to be a more effective novel treatment for periodontal regeneration.

\section{Dental-Tissue-Derived Stem Cells}

DPSCs have been shown to possess the potential to modulate immune function in a similar way to PDLSCs [7••]. Human DPSCs inhibited PBMNC proliferation following mitogen stimulation or in an allogeneic mixed lymphocyte reaction, which was mediated in part by soluble factors produced by activated PBMNCs. These observations are supported by other studies demonstrating the immunomodulatory effects of swine and human DPSCs on PBMNCs [45, 46]. Furthermore, SHED, which is derived from dental pulp tissue of exfoliated deciduous teeth, showed immunosuppressive effects on the proliferation of Th17 cells in vitro. Furthermore, SHED transplantation into SLE-like MRL/lpr mice improved SLE phenotypes (including renal problems), suggesting that SHED transplantation could be an effective approach for treating SLE disorders [47]. Recently, acetylsalicylic acid has been reported to improve SHED immunomodulation by elevating telomerase activity [48]. Systemic infusion of SHED ameliorates the osteoporotic phenotype in ovariectomized (OVX) mice by rescuing OVX-induced impairment of BMSCs, while also inhibiting osteoclastogenesis induced by activated T cell apoptosis in OVX mice via activation of the Fas-ligand-mediated Fas pathway [49]. It has also been reported that porcine and human SCAP suppressed T cell proliferation through an apoptosis-independent mechanism in vitro, while human DFCs stimulated with agonists of Toll-like receptor (TLR) 3 or TLR4 exhibited more effective suppression of PBMNC proliferation [46, 50, 51]. Because of their immunomodulatory functions, dental-tissue-derived stem cells are expected to be a good cell source for oral tissue engineering or autoimmune disease treatments. Among the different dental- 
tissue-derived stem cells, GMSCs have recently gained some attention because the gingiva is relatively easily accessible and can often be non-invasively obtained as a discarded sample. Human GMSCs have also been shown to possess a higher proliferation capacity but more limited differentiation potential and express cell surface molecule characteristic of MSCs, when compared to BMSCs [37]. Thus, GMSCs can be easily expanded ex vivo for cell-based clinical applications but may be restricted in their applications. Furthermore, GMSCs have also been reported to possess immunomodulatory capabilities, comparable to other MSC-like populations [37, 52]. GMSCs suppressed PBMNC proliferation and enhanced the expression of IL-10, IDO, inducible nitric oxide synthase, and cyclooxygenase 2 , all of which are immunosuppressive factors, in response to stimulation with the inflammatory cytokine, IFN- $\gamma$. Systemic infusion of GMSCs into an experimental colitis mouse model significantly ameliorated both the clinical and histopathological severity of the colonic inflammation mediated by the suppression of inflammatory infiltrates and inflammatory cytokine expression and inhibited regulatory $\mathrm{T}$ cell infiltration and IL-10 expression at the colonic sites [37]. Another study revealed that systemic infusion of hypoxiastimulated GMSCs into an excisional skin-wound-healing mouse model enhanced wound repair mediated by the suppression of inflammatory cells and pro-inflammatory cytokine expression, and an increase in IL-10 expression [52]. Although further studies are needed, GMSCs could be an attractive cell source for promoting tissue regeneration and recovery in autoimmune and inflammatory diseases.

\section{Periodontal Regeneration}

To effectively regenerate periodontal tissue that has been damaged and destroyed by periodontitis, cell transplantation methods based on the immunomodulatory properties of PDLSCs or MSCs have been examined in recent years.

\section{Allogeneic PDLSC Transplantation}

As already discussed herein, PDLSCs exhibit different characteristics in vivo compared with BMSCs and DPSCs. In a subcutaneous cell transplantation mouse model, PDLSC formed periodontal tissue-like tissue, including a PDL-like structure, while BMSCs formed bone-like tissue and DPSCs formed dentin/pulp complex-like tissue [6]. From these results, PDLSCs are considered to be an ideal cell source to regenerate periodontal tissue in contrast to MSCs derived from other dental tissues. However, it is often difficult to generate enough PDLSCs from one donor source because the growth and differentiation potentials of PDLSCs vary between donors. In addition, PDL samples are at risk of containing bacterial or fungal contaminations. Thus, utilizing a pool of well-characterized healthy PDLSCs from an allogeneic source represents an attractive option to generate enough cells for implantation with a defined regenerative capacity. Although the use of allogeneic cells have the potential for rejection by the host immune system, some studies have recently demonstrated positive results of allogeneic PDLSC transplantation for the regeneration of periodontal tissue.

In a normal rat periodontal fenestration defect model, allogeneic PDLSC transplantation with a scaffold promoted repair of periodontal defects by forming new bone, PDL, and cementum-like tissue, without showing any signs of inflammation [53].

Ding et al. demonstrated that in a procine periodontitis model, the transplantation of allogeneic PDLSC sheets regenerated periodontal tissue without stimulating immune rejection [40••]. In an allogeneic PDLSC transplantation group, the probing depth determined by clinical assessment, alveolar bone recovery assessed by computed tomography, and the observed regeneration of new bone, cementum, and periodontal ligament by histological examination were similar to autologous PDLSC sheet transplantation, whereas the HA/TCP alone and control groups were decreased in comparison. Furthermore, the analysis of T-cell-related immunological marker expression, routine blood and biochemical tests, and immunoglobulin tests in whole blood of cell-transplanted swine models showed no significant differences between allogeneic and autologous PDLSCs, suggesting that the allogenic cells did not induce immunological rejection. The same research group examined the use of allogeneic PDLSCs and DPSCs for bio-root regeneration in a porcine model [54]. It has been recently reported that allogeneic porcine SHED induced remarkable repair of periodontal tissue in a periodontitis model to a similar degree as allogeneic PDLSCs [55]. In an ovine periodontal fenestration defect model, histological examination indicated that transplantation of allogeneic PDLSCs combined with a scaffold displayed significantly greater new alveolar bone area, cementum length, and Sharpey's fiber thickness compared with the control [56 ]. In addition, allogeneic PDLSCs were well tolerated by recipient animals, as evidenced by the lack of inflammation, infection, or root exposure in all treatment groups. These studies are important for establishing and developing the therapeutic modality of allogeneic PDLSC transplantation for patients with severe periodontitis, where obtaining sufficient numbers of autologous cells is not clinically feasible.

\section{Anti-inflammatory Effects}

Owing to their immunomodulatory properties, PDLSCs are expected to be suitable cell sources for periodontal regeneration following periodontitis. However, Zhang et al. reported that BMSCs exhibited stronger osteogenic differentiation than PDLSCs stimulated with the inflammatory cytokine TNF- $\alpha$ in vitro, indicating that BMSCs might have better 
immunomodulatory properties in the local microenvironment compared with PDLSCs [57]. Allogeneic BMSC transplantation into a mouse periodontal defect model has been reported to regenerate bone, cementum, and PDL while integrating into all three newly formed tissues [58], and locally suppress the expression of inflammatory cytokines, including TNF- $\alpha$ and IL-1 [59]. These findings suggest that BMSC transplantation could also be feasible for periodontal regeneration, especially in alveolar bone regeneration under inflammatory conditions like those that occur after periodontal surgery for chronic periodontitis. However, BMSCs have been shown to predominantly form lamellae bone and bone marrow organs when transplanted in vivo and lack high expression of the transcription factor, Scleraxis, which may limit their capacity to form ligamentous fibers [6].

\section{Future Perspective}

In recent years, research in regenerative medicine using stem cells has developed at an accelerated rate with over 500 reported clinical trials utilizing different MSC-like populations for a wide range of clinical indications. To date, in the dental field, basic scientists, clinical investigators, and dentists have been investigating alternative effective treatments for regenerating periodontal tissue using MSCs, including PDLSCs. One of the main problems with using autologous PDLSCs for cell transplantation is the technical difficulty in isolating sufficient numbers of high-quality PDLSCs from a single patient because the growth and differentiation potentials of cells vary between individuals or cell pools. One way to address this problem is to establish a cell transplantation method using allogeneic PDLSCs as an alternative cell source. More recently, the Center for iPS Cell Research and Application (CiRA) in Japan have begun to provide induced pluripotent stem (iPS) cell banks available for regenerative medicine. As these iPS cell banks are generated from highfrequency homozygous human leukocyte antigen (HLA) donors, the transplantation of allogeneic cells differentiated from these iPS cell lines is believed to be less immunogenic [60]. Compared with iPS cells generated from the patient's own cells, utilizing cell banks has the advantage in that it significantly reduces time and costs for manufacturing iPS cells. Because of their immunomodulatory properties, allogeneic transplantation of PDLSCs might be a more feasible alternative stem cell source. Although further studies are required to investigate the efficacy and safety of allogeneic MSC therapies in human clinical trials, cryopreserved banks of allogeneic PDLSC of known HLA type may be an ideal therapeutic strategy for periodontal tissue regeneration for whole communities. Thus, the establishment of methods or the identification of factors to increase the yield and potency of PDLSCs is also of great importance.

\section{Conclusion}

In recent years, the therapeutic potential of PDLSCs has been explored in a number of studies. Currently, a clinical trial examining the use of human PDL cell sheets, which include PDLSCs, for transplantation in periodontal tissue regeneration, is ongoing in Japan [61]. PDLSCs possess a great potential for proliferation, self-renewal, and multidifferentiation and also exhibit immunomodulatory properties, which could have good potential for exhibiting anti-inflammatory effects and immune tolerance at the time of cell transplantation. Thus, autologous PDLSCs are now being considered as the principal cell of choice for tissue-engineering applications, while wellcharacterized PDLSCs from allogeneic sources might also represent an alternative approach in the future to compensate the potential weakness of autologous cell transplantation, which is the difficulty to generate a sufficient number of PDLSCs from a single donor. Although further studies are needed to establish a novel regenerative treatment using PDLSC transplantation, studies investigating their potential for clinical application are advancing slowly but steadily.

Acknowledgments This work was supported by Grants-in-Aid for Scientific Research from the Japan Society for the Promotion of Science (Project Nos. 26670826 and 15H05023), and the National Health \& Medical research Council of Australia (grant No 627143).

\section{Compliance with Ethics Guidelines}

Conflict of Interest Dr. Naohisa Wada, Dr. Atsushi Tomokiyo, and Dr. Stan Gronthos declare no potential conflicts of interest relevant to this article. Dr. P. Mark Bartold serves as a section editor for Current Oral Health Reports.

Human and Animal Rights and Informed Consent This article does not contain any studies with human or animal subjects performed by any of the authors.

\section{References}

Papers of particular interest, published recently, have been highlighted as:

- Of importance

- Of major importance

1. Gottlow J. Guided tissue regeneration using bioresorbable and nonresorbable devices: initial healing and long-term results. J Periodontol. 1993;64(11 Suppl):1157-65.

2. Venezia E, Goldstein M, Boyan BD, Schwartz Z. The use of enamel matrix derivative in the treatment of periodontal defects: a literature review and meta-analysis. Crit Rev Oral Biol Med. 2004;15(6): 382-402.

3. Lynch SE, Wisner-Lynch L, Nevins M, Nevins ML. A new era in periodontal and periimplant regeneration: use of growth-factor enhanced matrices incorporating rhPDGF. Compend Contin Educ Dent. 2006;27(12):672-8. quiz 679-80. 
4. Murakami, S., Periodontal tissue regeneration by signaling molecule(s): what role does basic fibroblast growth factor (FGF-2) have in periodontal therapy? Periodontol 2000, 2011. 56(1): 188-208.

5. Gronthos S, Mankani M, Brahim J, Robey PG, Shi S. Postnatal human dental pulp stem cells (DPSCs) in vitro and in vivo. Proc Natl Acad Sci U S A. 2000;97(25):13625-30.

6. Seo BM, Miura M, Gronthos S, Bartold PM, Batouli S, Brahim J, et al. Investigation of multipotent postnatal stem cells from human periodontal ligament. Lancet. 2004;364(9429):149-55.

7.. Wada N, Menicanin D, Shi S, Bartold PM, Gronthos S. Immunomodulatory properties of human periodontal ligament stem cells. J Cell Physiol. 2009;219(3):667-76. This publication was the first to demonstrate that human PDLSCs possess immunomodulatory property.

8. Tse WT, Pendleton JD, Beyer WM, Egalka MC, Guinan EC. Suppression of allogeneic T-cell proliferation by human marrow stromal cells: implications in transplantation. Transplantation. 2003;75(3):389-97.

9. Duffy MM, Ritter T, Ceredig R, Griffin MD. Mesenchymal stem cell effects on T-cell effector pathways. Stem Cell Res Ther. 2011;2(4):34.

10. Cassatella MA, Mosna F, Micheletti A, Lisi V, Tamassia N, Cont C, et al. Toll-like receptor-3-activated human mesenchymal stromal cells significantly prolong the survival and function of neutrophils. Stem Cells. 2011;29(6):1001-11.

11. Corcione A, Benvenuto F, Ferretti E, Giunti D, Cappiello V, Cazzanti F, et al. Human mesenchymal stem cells modulate B-cell functions. Blood. 2006;107(1):367-72.

12. Jiang XX, Zhang Y, Liu B, Zhang SX, Wu Y, Yu XD, et al. Human mesenchymal stem cells inhibit differentiation and function of monocyte-derived dendritic cells. Blood. 2005;105(10):4120-6.

13. Spaggiari GM, Capobianco A, Abdelrazik H, Becchetti F, Mingari MC, Moretta L. Mesenchymal stem cells inhibit natural killer-cell proliferation, cytotoxicity, and cytokine production: role of indoleamine 2,3-dioxygenase and prostaglandin E2. Blood. 2008;111(3):1327-33.

14. Eggenhofer E, Hoogduijn MJ. Mesenchymal stem cell-educated macrophages. Transplant Res. 2012;1(1):12.

15. Di Nicola M, Carlo-Stella C, Magni M, Milanesi M, Longoni PD, Matteucci P, et al. Human bone marrow stromal cells suppress Tlymphocyte proliferation induced by cellular or nonspecific mitogenic stimuli. Blood. 2002;99(10):3838-43.

16. Meisel R, Zibert A, Laryea M, Gobel U, Daubener W, Dilloo D. Human bone marrow stromal cells inhibit allogeneic T-cell responses by indoleamine 2,3-dioxygenase-mediated tryptophan degradation. Blood. 2004;103(12):4619-21.

17. Aggarwal S, Pittenger MF. Human mesenchymal stem cells modulate allogeneic immune cell responses. Blood. 2005;105(4):181522.

18. Sato K, Ozaki K, Oh I, Meguro A, Hatanaka K, Nagai T, et al. Nitric oxide plays a critical role in suppression of T-cell proliferation by mesenchymal stem cells. Blood. 2007;109(1):228-34.

19. Bouffi C, Bony C, Courties G, Jorgensen C, Noel D. IL-6dependent PGE2 secretion by mesenchymal stem cells inhibits local inflammation in experimental arthritis. PLoS One. 2010;5(12), e14247.

20. Volarevic V, Al-Qahtani A, Arsenijevic N, Pajovic S, Lukic ML. Interleukin-1 receptor antagonist (IL-1Ra) and IL-1Ra producing mesenchymal stem cells as modulators of diabetogenesis. Autoimmunity. 2010;43(4):255-63.

21. Chabannes D, Hill M, Merieau E, Rossignol J, Brion R, Soulillou $\mathrm{JP}$, et al. A role for heme oxygenase-1 in the immunosuppressive effect of adult rat and human mesenchymal stem cells. Blood. 2007;110(10):3691-4.
22. Nasef A, Mathieu N, Chapel A, Frick J, Francois S, Mazurier C, et al. Immunosuppressive effects of mesenchymal stem cells: involvement of HLA-G. Transplantation. 2007;84(2):231-7.

23. Bartholomew A, Sturgeon C, Siatskas M, Ferrer K, McIntosh K, Patil S, et al. Mesenchymal stem cells suppress lymphocyte proliferation in vitro and prolong skin graft survival in vivo. Exp Hematol. 2002;30(1):42-8.

24. Lu X, Liu T, Gu L, Huang C, Zhu H, Meng W, et al. Immunomodulatory effects of mesenchymal stem cells involved in favoring type $2 \mathrm{~T}$ cell subsets. Transpl Immunol. 2009;22(12):55-61.

25. Sun L, Akiyama K, Zhang H, Yamaza T, Hou Y, Zhao S, et al. Mesenchymal stem cell transplantation reverses multiorgan dysfunction in systemic lupus erythematosus mice and humans. Stem Cells. 2009;27(6):1421-32.

26. Augello A, Tasso R, Negrini SM, Cancedda R, Pennesi G. Cell therapy using allogeneic bone marrow mesenchymal stem cells prevents tissue damage in collagen-induced arthritis. Arthritis Rheum. 2007;56(4):1175-86.

27. Zappia E, Casazza S, Pedemonte E, Benvenuto F, Bonanni I, Gerdoni E, et al. Mesenchymal stem cells ameliorate experimental autoimmune encephalomyelitis inducing T-cell anergy. Blood. 2005;106(5):1755-61.

28. Cohen JA. Mesenchymal stem cell transplantation in multiple sclerosis. J Neurol Sci. 2013;333(1-2):43-9.

29. Le Blanc K, Rasmusson I, Sundberg B, Gotherstrom C, Hassan M, Uzunel $\mathrm{M}$, et al. Treatment of severe acute graft-versus-host disease with third party haploidentical mesenchymal stem cells. Lancet. 2004;363(9419):1439-41.

30. Le Blanc K, Frassoni F, Ball L, Locatelli F, Roelofs H, Lewis I, et al. Mesenchymal stem cells for treatment of steroid-resistant, severe, acute graft-versus-host disease: a phase II study. Lancet. 2008;371(9624):1579-86.

31. Sharma RR, Pollock K, Hubel A, McKenna D. Mesenchymal stem or stromal cells: a review of clinical applications and manufacturing practices. Transfusion. 2014;54(5):1418-37.

32. Liang J, Zhang H, Hua B, Wang H, Lu L, Shi S, et al. Allogenic mesenchymal stem cells transplantation in refractory systemic lupus erythematosus: a pilot clinical study. Ann Rheum Dis. 2010;69(8):1423-9.

33. Sun L, Wang D, Liang J, Zhang H, Feng X, Wang H, et al. Umbilical cord mesenchymal stem cell transplantation in severe and refractory systemic lupus erythematosus. Arthritis Rheum. 2010;62(8):2467-75.

34. Miura M, Gronthos S, Zhao M, Lu B, Fisher LW, Robey PG, et al. SHED: stem cells from human exfoliated deciduous teeth. Proc Natl Acad Sci U S A. 2003;100(10):5807-12.

35. Sonoyama W, Liu Y, Fang D, Yamaza T, Seo BM, Zhang C, et al. Mesenchymal stem cell-mediated functional tooth regeneration in swine. PLoS One. 2006;1, e79.

36. Morsczeck C, Gotz W, Schierholz J, Zeilhofer F, Kuhn U, Mohl C, et al. Isolation of precursor cells (PCs) from human dental follicle of wisdom teeth. Matrix Biol. 2005;24(2):155-65.

37. Zhang Q, Shi S, Liu Y, Uyanne J, Shi Y, Le AD. Mesenchymal stem cells derived from human gingiva are capable of immunomodulatory functions and ameliorate inflammation-related tissue destruction in experimental colitis. J Immunol. 2009;183(12):7787-98.

38. Kim HS, Kim KH, Kim SH, Kim YS, Koo KT, Kim TI, et al. Immunomodulatory effect of canine periodontal ligament stem cells on allogenic and xenogenic peripheral blood mononuclear cells. J Periodontal Implant Sci. 2010;40(6):265-70.

39. Dokic J, Tomic S, Cerovic S, Todorovic V, Rudolf R, Colic M. Characterization and immunosuppressive properties of mesenchymal stem cells from periapical lesions. J Clin Periodontol. 2012;39(9):807-16. 
40.• Ding G, Liu Y, Wang W, Wei F, Liu D, Fan Z, et al. Allogeneic periodontal ligament stem cell therapy for periodontitis in swine. Stem Cells. 2010;28(10):1829-38. This publication was the first to demonstrate allogeneic swine PDLSC transplantation into periodontitis model to regenerate periodontal tissue.

41. Tang R, Wei F, Wei L, Wang S, Ding G. Osteogenic differentiated periodontal ligament stem cells maintain their immunomodulatory capacity. J Tissue Eng Regen Med. 2014;8(3):226-32.

42. Liu O, Xu J, Ding G, Liu D, Fan Z, Zhang C, et al., Periodontal ligament stem cells regulate B lymphocyte function via programmed cell death protein 1. Stem Cells. 2013;31(7):1371-82. This publication was the first to demonstrate that human PDLSCs suppress B lymphocyte function.

43. Liu D, Xu J, Liu O, Fan Z, Liu Y, Wang F, et al. Mesenchymal stem cells derived from inflamed periodontal ligaments exhibit impaired immunomodulation. J Clin Periodontol. 2012;39(12):1174-82.

44. Park JC, Kim JM, Jung IH, Kim JC, Choi SH, Cho KS, et al. Isolation and characterization of human periodontal ligament (PDL) stem cells (PDLSCs) from the inflamed PDL tissue: in vitro and in vivo evaluations. J Clin Periodontol. 2011;38(8):721-31.

45. Tang R, Ding G. Swine dental pulp stem cells inhibit T-cell proliferation. Transplant Proc. 2011;43(10):3955-9.

46. Tomic S, Djokic J, Vasilijic S, Vucevic D, Todorovic V, Supic G, et al. Immunomodulatory properties of mesenchymal stem cells derived from dental pulp and dental follicle are susceptible to activation by toll-like receptor agonists. Stem Cells Dev. 2011;20(4): 695-708.

47. Yamaza T, Kentaro A, Chen C, Liu Y, Shi Y, Gronthos S, et al. Immunomodulatory properties of stem cells from human exfoliated deciduous teeth. Stem Cell Res Ther. 2010;1(1):5.

48. Liu Y, Chen C, Liu S, Liu D, Xu X, Chen X, et al. Acetylsalicylic acid treatment improves differentiation and immunomodulation of SHED. J Dent Res. 2015;94(1):209-18.

49. Liu Y, Wang L, Liu S, Liu D, Chen C, Xu X, et al. Transplantation of SHED prevents bone loss in the early phase of ovariectomyinduced osteoporosis. J Dent Res. 2014;93(11):1124-32.

50. Ding G, Liu Y, An Y, Zhang C, Shi S, Wang W, et al. Suppression of $\mathrm{T}$ cell proliferation by root apical papilla stem cells in vitro. Cells Tissues Organs. 2010;191(5):357-64.
51. Ding G, Wang W, Liu Y, An Y, Zhang C, Shi S, et al. Effect of cryopreservation on biological and immunological properties of stem cells from apical papilla. J Cell Physiol. 2010;223(2):415-22.

52. Jiang CM, Liu J, Zhao JY, Xiao L, An S, Gou YC, et al. Effects of hypoxia on the immunomodulatory properties of human gingivaderived mesenchymal stem cells. J Dent Res. 2015;94(1):69-77.

53. Han J, Menicanin D, Marino V, Ge S, Mrozik K, Gronthos S, et al. Assessment of the regenerative potential of allogeneic periodontal ligament stem cells in a rodent periodontal defect model. J Periodontal Res. 2014;49(3):333-45.

54. Wei F, Song T, Ding G, Xu J, Liu Y, Liu D, et al. Functional tooth restoration by allogeneic mesenchymal stem cell-based bio-root regeneration in swine. Stem Cells Dev. 2013;22(12):1752-62.

55. Fu X, Jin L, Ma P, Fan Z, Wang S. Allogeneic stem cells from deciduous teeth in treatment for periodontitis in miniature swine. $\mathrm{J}$ Periodontol. 2014;85(6):845-51.

56. Mrozik KM, Wada N, Marino V, Richter W, Shi S, Wheeler DL, et al. Regeneration of periodontal tissues using allogeneic periodontal ligament stem cells in an ovine model. Regen Med. 2013;8(6): 711-23. This publication was the first to demonstrate allogeneic ovine PDLSC transplantation into periodontitis model to regenerate periodontal tissue.

57. Zhang J, Li ZG, Si YM, Chen B, Meng J. The difference on the osteogenic differentiation between periodontal ligament stem cells and bone marrow mesenchymal stem cells under inflammatory microenviroments. Differentiation. 2014;88(4-5):97-105.

58. Yang Y, Rossi FMV, Putnins EE. Periodontal regeneration using engineered bone marrow mesenchymal stromal cells. Biomaterials. 2010;31(33):8574-82.

59. Du J, Shan Z, Ma P, Wang S, Fan Z. Allogeneic bone marrow mesenchymal stem cell transplantation for periodontal regeneration. J Dent Res. 2014;93(2):183-8.

60. Okita K, Matsumura Y, Sato Y, Okada A, Morizane A, Okamoto S, et al. A more efficient method to generate integration-free human iPS cells. Nat Methods. 2011;8(5):409-12.

61. Yoshida T, Washio K, Iwata T, Okano T, Ishikawa I. Current status and future development of cell transplantation therapy for periodontal tissue regeneration. Int J Dent. 2012;2012:307024. 